\title{
CARACTERIZACIÓN DE LAS PRÁCTICAS DE GESTIÓN DE ACTUALIZACIÓN E INNOVACIÓN TECNOLÓGICA EN EL ÁREA DE PRODUCCIÓN DE ORGANIZACIONES DE LA INDUSTRIA GRÁFICA EN COLOMBIA
} CHARACTERIZATION WORK ABOUTMANAGEMENT PRACTICES OF TECHNOLOGY UPGRADE AND INNOVATION IN PRODUCTION AREA OF ORGANIZATIONS IN THE GRAPHIC INDUSTRY IN COLOMBIA CARACTERIZAÇÃO DAS PRÁTICAS DE GESTÃO DE PROCESSOS DE ATUALIZAÇÃO E INOVAÇÃO TECNOLÓGICA NA ÁREA DE PRODUÇÃO DAS ORGANIZAÇÕES DA INDÚSTRIA GRÁFICA NA COLÔMBIA

\section{María Victoria Vásquez-Rodríguez}

Mestre em Administração pela Universidade Nacional da Colômbia.

\section{RESUMEN}

Con base en una muestra de organizaciones de la industria gráfica en Colombia se recolectó información sobre cómo estas empresas interactúan con su entorno y cómo el empresario toma las decisiones para enfrentar los retos de actualización e innovación tecnológica. Se siguieron las pautas de una investigación de tipo cualitativo. Esta caracterización pretende aportar a la identificación de elementos que pudieran servir de insumo para el diseño de políticas o iniciativas de organismos públicos o privados que apoyan el sector. En la muestra se destacan algunas prácticas: asociarse con organizaciones que promueven innovación basada en tecnología, implementar procedimientos formales para gestión de proyectos de actualización e innovación y apoyar desde la administración capacitación en temas de gestión e innovación. Se concluye que promover y fortalecer alianzas entre integrantes de la Industria y con entidades de apoyo debería dinamizar el sector para generar e implementar iniciativas de mejora en competitividad, productividad e innovación.

Palabras clave: Caracterización. Cambio tecnológico. Industria gráfica. Gestión. Innovación.

\section{ABSTRACT}

Based on a sample of organizations that are part of the graphic industry in Colombia, we collect information on how these companies interact with their environment and how entrepreneurs make decisions in order to face the challenges of technological upgrade and innovation. The guidelines of a qualitative research were followed as methodology. This characterization work intends to contribute to the identification of elements that could serve as input for the design of policies or initiatives in public or private organizations that support the sector. The present work highlights certain practices by the entrepreneurs included in the sample: partnering with organizations that promote technology-based innovation; implementing formal procedures to manage the technological upgrade and innovation; and supporting training programs for personnel in the subjects of management and innovation. It is concluded that promoting and strengthening alliances between members of the Industry and support entities should dynamize the sector to generate and implement improvement initiatives in competitiveness, productivity and innovation.

Keywords: Characterization. Technological change. Graphic industry. Innovation. Management.

\section{RESUMO}

Baseado em uma amostra de organizações que fazem parte da indústria gráfica da Colômbia, coletamos informações sobre como essas empresas interagem com seu meio e como os empresários tomam decisões para enfrentar os desafios da atualização e inovação tecnológica. Seguimos as diretrizes de uma pesquisa qualitativa como metodologia. Esse trabalho de caracterização pretende contribuir para a identificação de elementos que podem servir de base para projetos políticos, iniciativas públicas ou privadas que apoiam o setor. O presente trabalho destaca algumas práticas dos empresários dessa amostra: parceria com organizações que promovem a inovação baseada em tecnologia; realizando procedimentos formais para gerenciar a atualização tecnológica e a inovação; apoiando programas de treinamento para equipe em assuntos de gestão e inovação. Conclui-se que promover e fortalecer alianças entre os membros da Indústria e entidades de apoio deve dinamizar o setor para gerar e realizar iniciativas de melhoria em competitividade, produtividade e inovação.

Palavras-chave: Caracterização. Mudanças tecnológicas. Indústria gráfica. Inovação. Gestão. 


\section{INTRODUCCIÓN}

Día a día las organizaciones de la industria de la comunicación gráfica en Colombia (ICGC) ${ }^{1}$ se enfrentan a nuevos retos de actualización tecnológica en el área de producción, pues a partir de los avances científicos y técnicos de las últimas décadas está disponible gran cantidad de nuevas herramientas para la elaboración de productos gráficos.

Así mismo, los avances en tecnologías de la información y la comunicación (TIC) han impulsado el desarrollo de nuevas maneras de presentar la información (libro electrónico, contenido multimedia, etc.), como respuesta a la aparición de diversos dispositivos electrónicos, como smartphones o tablets, entre otros.

La mejora en productividad es el beneficio percibido por las organizaciones al realizar inversión en adquisición de maquinaria y equipo ${ }^{2}$, y se refleja en la disminución de costos ocultos y tiempos de alistamiento. Pero alrededor de este efecto evidente, subyacen las prácticas de las organizaciones en cuanto a asimilación ${ }^{3}$ de tecnología: desde las fuentes de información y financiación, pasando por la capacitación y entrenamiento en su uso, hasta las posibles adaptaciones o mejoras según las necesidades de producción.

La ICGC se caracteriza por estar conformada por un alto porcentaje de MiPyME (más del 90\%), situación que implica la coexistencia de gran

\footnotetext{
1 Se reconoce al conjunto de actividades de edición, impresión y relacionadas, como "industria de la comunicación gráfica" (ANDIGRAF, 1994, p. VII).

2 Es uno de los tipos de actividades de ciencia, tecnología e innovación $(\mathrm{ACTI})$, enumerados en encuestas de innovación DANE e indicadores de ciencia y tecnología OCyT. En el Manual de Bogotá aparece como "adquisición de tecnología incorporada al capital" (RICYT et al., 2001 pp. 38-39). 3 Entendido como la comprensión del nuevo aprendizaje y su incorporación a conocimientos previos.
}

variedad de prácticas de gestión. Así mismo, por la incidencia de aspectos socioculturales y de acceso a recursos, es común encontrar que las MiPyME funcionan cumpliendo sus procesos de manera "informal" , más interesadas en sobrellevar "el día a día" que garantice su supervivencia en el mercado.

Por lo anterior, la pregunta que guía este trabajo es: ¿cómo enfrentan las organizaciones de la ICGC los retos de la gestión de procesos de actualización e innovación tecnológica en el área de producción?

La presente caracterización de prácticas de gestión de algunos empresarios de la Industria pretende aportar la identificación de factores que pudieran afectar o influir en la realización de proyectos de actualización e innovación tecnológica. Cómo estas prácticas de gestión podrían promover el avance y desarrollo del sector, y cómo podrían contribuir al desarrollo de estrategias para responder a las nuevas exigencias del mercado.

Se parte de reconocer las restricciones que se tuvieron para la conformación de la muestra y recolección de información. Sin embargo, se estima que se cumple con el objetivo de identificar y describir algunas prácticas de algunos empresarios de la ICGC frente al cambio tecnológico que vive el sector.

\section{INDUSTRIA DE LA COMUNICACIÓN GRÁFICA}

La evolución tecnológica del sector ha modificado el entorno y el modelo de negocios tradicional, lo que trajo como consecuencia la desaparición o disminución del mercado de una importante cantidad de productos impresos. Así mismo, en el 
contexto del ascenso de los medios digitales, el factor crítico para el crecimiento a largo plazo de las organizaciones del sector es «la capacidad de gestionar la innovación para adaptarse a las necesidades de los clientes» (PTP, 2016a, pp. 14-15).

Para el periodo 2009-2014 las exportaciones de la ICG en el mundo presentaron una TACC ${ }^{4}$ de 5,07\%, la ICG en América Latina 4,66\% y la ICGC -8,94\%.

La participación de la región respecto a las exportaciones mundiales pasó de 3,12\% en 2009 a 3,06\% en 2014. La participación de Colombia ha ido decreciendo, pasando de $0,37 \%$ en 2009 a 0,18\% en 2014 (Figura 1).

Figura 1. Exportaciones mundiales ICG 2009-2014 (Miles de dólares)

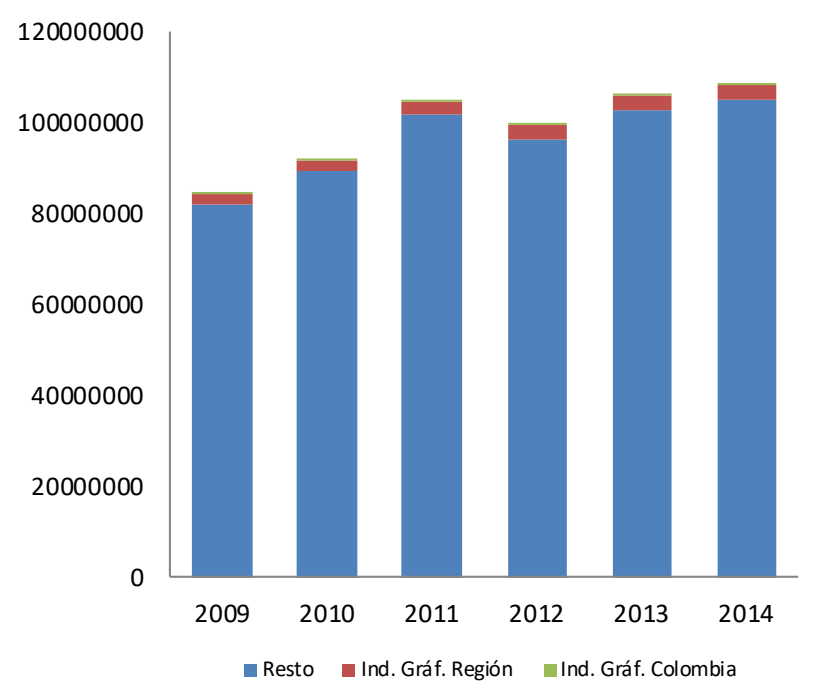

Fuente: PTP (2016a, p.58) a partir de UN COMTRADE.

Para el año 2014 en la ICG de América Latina, México participó con 46,94\% de las exportaciones, seguido por Brasil (9,25\%). La ICGC pasó del segundo lugar en 2009 (11,85\%) al quinto en 2014 (5,91\%) (Figura 2).

4 Tasa de crecimiento anual compuesto, en inglés Compound Annual Growth Rate (CAGR).
Figura 2. Exportaciones ICG América Latina 2009-2014 (Miles de dólares)

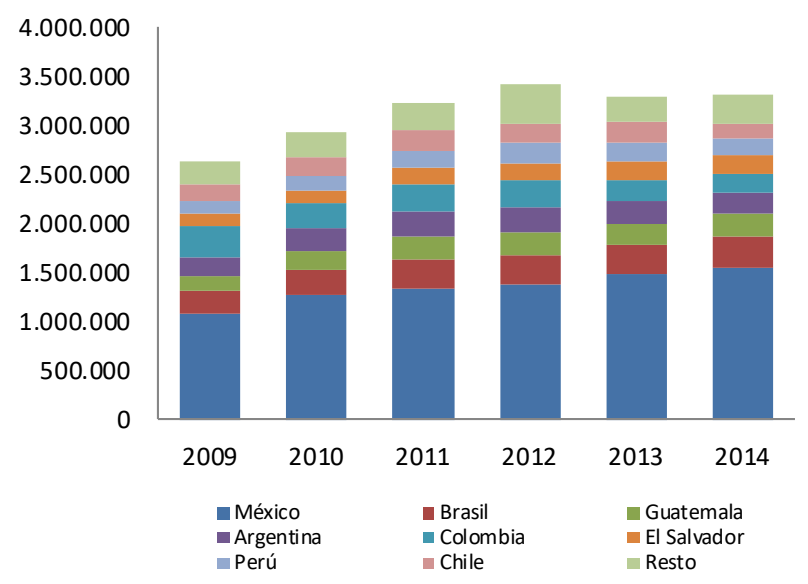

Fuente: PTP (2016a, p. 60) a partir de UN COMTRADE.

En el periodo 2009-2014 se observa que las exportaciones del sector presentaron una TACC de $-8,99 \%$ frente a $-0,12 \%$ de las manufacturas y $10,78 \%$ de las exportaciones del país. Respecto al total, las exportaciones de la ICGC pasaron de representar el 0,96\% en 2009 al 0,36\% en 2014 (Figura 3).

Figura 3. Exportaciones Colombia, Industria manufacturera e ICGC 2009-2014 (Millones de Dólares FOB)

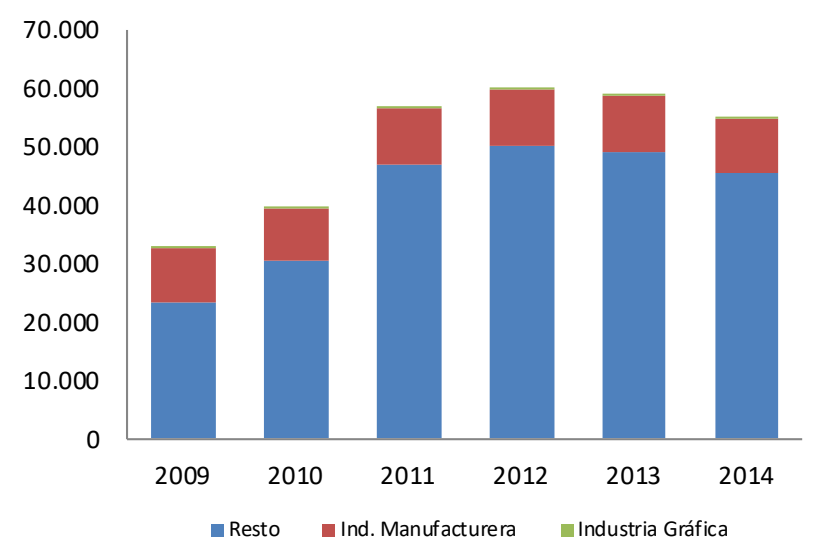

Fuente: DANE (2017a) y PTP (2016a, p. 61).

\subsection{VARIABLES PRINCIPALES ICGC}

En el año 2015, la EAM identificó 525 establecimientos dedicados a las actividades de impre- 
sión y de servicios relacionados con la impresión ${ }^{5}$. De esta cifra la participación de la MiPyME correspondió a 96,88\%. La ICGC se concentra en tres regiones: Bogotá (49,33\%), Antioquia (20,76\%), y Valle (12,76\%). Respecto a la producción bruta de la ICGC en el año 2015, la participación de la MiPyME correspondió a 67,71\%. La producción se concentra en tres regiones: Bogotá (49,58\%), Valle $(23,44 \%)$, y Antioquia $(16,86 \%)$

Con base en registros de 2015 de las principales cámaras de comercio y según cál- culos de Andigraf, la ICGC «comprende aproximadamente 6.700 establecimientos. En 2014, su valor agregado superó los $\$ 2$ billones de pesos, exportó 195 millones USD FOB, y empleó 61.057 trabajadores directos». Además de estas 6.700 unidades se resalta la alta concentración en MiPyME (99,6\%). La gran empresa a pesar de contar con solo un $0,4 \%$ en el número de establecimientos, alcanza una participación de $85,9 \%$ en activos y $85,6 \%$ en ventas (PTP, 2016a, pp. 8, 34).

Tabla 1. Características generales organizaciones ICGC

\begin{tabular}{|c|c|c|}
\hline & Gran empresa & MiPyME \\
\hline Constitución & $\begin{array}{l}\text { La mayoría están constituidas como } \\
\text { sociedades. }\end{array}$ & $\begin{array}{l}\text { En su mayoría son organizaciones } \\
\text { unipersonales o de carácter familiar. }\end{array}$ \\
\hline Formalidad & $\begin{array}{l}\text { Al ser pocas empresas en el país, los } \\
\text { organismos de vigilancia del Estado tienen } \\
\text { mayor control sobre el ejercicio de estas } \\
\text { organizaciones. }\end{array}$ & $\begin{array}{l}\text { Niveles altos de informalidad empresarial, } \\
\text { informalidad laboral, e informalidad de } \\
\text { producción. }\end{array}$ \\
\hline Actividad & $\begin{array}{l}\text { Su estructura organizacional, física y } \\
\text { tecnológica permite integrar gran parte de las } \\
\text { actividades de la cadena de valor del sector. }\end{array}$ & $\begin{array}{l}\text { Manufactura de productos impresos, en } \\
\text { mayor proporción corresponde al proceso de } \\
\text { impresión. }\end{array}$ \\
\hline Producción & $\begin{array}{l}\text { Volumen alto, cuentan con tecnologías de alta } \\
\text { velocidad y capacidad de producción. }\end{array}$ & $\begin{array}{l}\text { Volumen bajo a medio, según tamaño } \\
\text { organización e infraestructura física y } \\
\text { tecnológica. }\end{array}$ \\
\hline $\begin{array}{l}\text { Oferta de valor } \\
\text { agregado }\end{array}$ & $\begin{array}{l}\text { Media a alta, ante los cambios tecnológicos y } \\
\text { las nuevas condiciones de los mercados, han } \\
\text { ampliado y diversificado sus portafolios de } \\
\text { productos, incluyendo servicios adicionales a } \\
\text { impresión. }\end{array}$ & $\begin{array}{l}\text { Baja, lo que sumado al hecho de que la } \\
\text { impresión es una actividad en el mercado de } \\
\text { los commodities }{ }^{6} \text {, conlleva altos niveles de } \\
\text { informalidad con el fin de bajar costos para } \\
\text { competir ("guerra de precios"). }\end{array}$ \\
\hline Mercado & $\begin{array}{l}\text { Alta capacidad de gestión y respuesta } \\
\text { eficiente y oportuna a las demandas de los } \\
\text { mercados nacionales e internacionales. }\end{array}$ & $\begin{array}{l}\text { En su mayoría se dedican a atender al } \\
\text { mercado local. }\end{array}$ \\
\hline $\begin{array}{l}\text { Capital de } \\
\text { trabajo }\end{array}$ & $\begin{array}{l}\text { Alto, además de facilidad en el acceso a } \\
\text { fuentes externas de financiación. }\end{array}$ & $\begin{array}{l}\text { Medio a bajo, sumado a la dificultad para } \\
\text { acceder a fuentes externas de financiación. }\end{array}$ \\
\hline
\end{tabular}

5 Para dar uniformidad a la información del DANE para la ICGC en el periodo 2009-2015 se utilizaron únicamente cifras de las actividades de impresión y servicios relacionados, contenidos en la EAM.

6 Se considera la impresión como actividad del mercado de los commodities (también llamados bienes básicos o genéricos), puesto que en sí misma no cuenta con mayor valor agregado, además de ser insumo para elaboración de otros bienes. 


\begin{tabular}{|c|c|c|}
\hline Gestión & $\begin{array}{l}\text { Estructura organizacional de complejidad } \\
\text { media-alta. División de tareas administrativas } \\
\text { y de producción en distintas áreas. Presencia } \\
\text { de instrumentos modernos de gestión. }\end{array}$ & $\begin{array}{l}\text { En la mayoría de MiPyME estas tareas son } \\
\text { realizadas por el gerente/propietario. No } \\
\text { hay procesos de planeación: "se apagan } \\
\text { incendios" y se va resolviendo el "día a día". } \\
\text { Ausencia de instrumentos modernos de } \\
\text { gestión. }\end{array}$ \\
\hline $\begin{array}{c}\text { Entorno } \\
\text { institucional }\end{array}$ & $\begin{array}{l}\text { Vínculo e interacción fuerte, acceso a } \\
\text { programas de apoyo gubernamentales. }\end{array}$ & $\begin{array}{l}\text { Vínculo e interacción débil, baja integración } \\
\text { con la academia y/o centros de desarrollo } \\
\text { tecnológico, bajo acceso a programas de } \\
\text { apoyo gubernamentales. }\end{array}$ \\
\hline Asociatividad $^{7}$ & Alto nivel de agremiación. & $\begin{array}{l}\text { Bajo nivel de agremiación }{ }^{8} \text {, tendencia al } \\
\text { individualismo, desconfianza (a nivel de } \\
\text { pares, y del entorno institucional). }\end{array}$ \\
\hline $\begin{array}{l}\text { Recursos } \\
\text { humanos }\end{array}$ & $\begin{array}{l}\text { Personal especializado y operarios } \\
\text { calificados con capacidad de responder a las } \\
\text { necesidades y expectativas de productividad } \\
\text { de la organización. }\end{array}$ & $\begin{array}{l}\text { Alta rotación del personal debido a la } \\
\text { informalidad laboral. Personal administrativo } \\
\text { y de producción con bajos niveles de } \\
\text { formación y capacitación. }\end{array}$ \\
\hline $\begin{array}{l}\text { Infraestructura } \\
\text { tecnológica }\end{array}$ & $\begin{array}{l}\text { Media a alta, base tecnológica moderna, de } \\
\text { alta velocidad y alta capacidad de producción. } \\
\text { Alta capacidad de adaptación a los cambios } \\
\text { tecnológicos del sector. }\end{array}$ & $\begin{array}{l}\text { Baja a media, en varias organizaciones la } \\
\text { base tecnológica es antigua, existe rezago } \\
\text { tecnológico debido al uso de maquinaria } \\
\text { obsoleta o a la compra en el mercado del } \\
\text { usado. }\end{array}$ \\
\hline $\begin{array}{l}\text { Inversión en } \\
\text { tecnología }\end{array}$ & $\begin{array}{l}\text { Incorporación permanente de las tecnologías } \\
\text { de punta (blanda y dura). } \\
\text { Amplia implementación y apropiación TIC. } \\
\text { Implementación de software que facilita la } \\
\text { gestión administrativa y de producción. }\end{array}$ & $\begin{array}{l}\text { Baja, preferencia de inversiones en } \\
\text { tecnologías "duras" puesto que se perciben } \\
\text { efectos inmediatos en productividad y } \\
\text { calidad. } \\
\text { Dificultades en implementación y baja } \\
\text { apropiación de TIC. }\end{array}$ \\
\hline $\begin{array}{l}\text { Gestión } \\
\text { innovación }\end{array}$ & $\begin{array}{l}\text { Implementación de sistemas de gestión de la } \\
\text { innovación. }\end{array}$ & $\begin{array}{l}\text { Ausencia de elementos formales de } \\
\text { gestión de la innovación. Preferencia por } \\
\text { "colombianización" de productos y procesos } \\
\text { del exterior. }\end{array}$ \\
\hline
\end{tabular}

Fuentes: SENA (2013), PTP (2009, 2013, 2016a), PÉREZ et al. (2014), ROBAYO (2015), BRICEÑO (2012), MALAVER (2002a, 2002b).

7 Andigraf agrupa especialmente a las grandes empresas. Las cooperativas agrupan organizaciones PyME del sector (SENA, 2013, p. 22).

8 Pertenecer a una asociación o cooperativa exige documentación legal al día y supone una cuota de afiliación, requisitos que muchos empresarios no están dispuestos o no tienen posibilidad de cumplir. 


\section{INNOVACIÓN EN PAÍSES EN DESARROLLO}

La competitividad en los países en desarrollo se basa en la explotación de recursos naturales y oferta de mano de obra barata y no en la eficiencia o diferenciación. Esto traduce innovación informal y baja inversión en I+D. Así mismo, la actividad de innovación a largo plazo se ve limitada por la incertidumbre del comportamiento macroeconómico, por lo que la debilidad en vínculos entre ciencia y empresa "obstaculizan la capacidad de las empresas para resolver los problemas (vinculados a la tecnología) y las empuja hacia soluciones que se basan principalmente en la adquisición de tecnologías incorporadas» (OCDE, 2006, pp. 156-158).

La preponderancia «de modos de innovar (adopción y adaptación), actividades de innovación (compra de maquinaria y equipo, ingeniería, diseño) y actores (marketing) distintos a la $I+D »$ tiene su origen en el patrón de desarrollo de industrias que no son technology push, sino demand pull o design driven. Por tanto, las políticas de innovación deberían promover el desarrollo de las capacidades de absorción, puesto que se propiciaría el uso creativo de las nuevas tecnologías «y avanzar de la adopción a la adaptación, y de esta a la creación» (MALAVER Y VARGAS, 2013, p. 564).

\subsection{OPORTUNIDADES EN “OTROS MODOS DE INNOVAR"}

En los países en desarrollo el aparato productivo se compone principalmente por empresas que aun sin tener base tecnológica, llevan a cabo esfuerzos tecnológicos «adaptando y mejorando lo ya existente y sin necesariamente aventurarse en ámbitos tecnológicamente más complejos, más inciertos y más cercanos a la frontera tecnológica mundial» (DINI; STUMPO, 2011, p. 90).

Actividades no basadas en I+D (por ejemplo diseño, uso de tecnología avanzada, formación o vigilancia tecnológica) tienen el potencial de contribuir a las organizaciones para que puedan obtener innovaciones pues «facilitan la adopción de tecnología, favorecen la introducción de cambios incrementales a los productos y procesos existentes, permiten combinar el conocimiento existente de forma distinta o, incluso, agilizan la imitación a través de la ingeniería inversa» (SANTAMARÍA et al., 2009, p. 102).

Las actividades más extendidas en las empresas que innovan mediante la adopción son compra de maquinaria y equipos, así como la «ingeniería requerida para operarlas». Así mismo la adaptación, capacitación, diseño e ingeniería son las «que permiten alcanzar innovaciones incrementales de carácter adaptativo; y en las que crean nuevos productos, la I+D y la capacitación, que conllevan aprendizaje tecnológico y desarrollo de la creatividad» (MALAVER Y VARGAS, 2012, p. 158).

\subsection{ENCUESTAS DE INNOVACIÓN ICGC}

El Manual de Bogotá introduce el concepto de "Gestión de la actividad innovadora", que incluye la innovación en sentido estricto y también al conjunto de actividades de innovación que trata el Manual de Oslo. Estas actividades son las acciones para «poner en práctica conceptos, ideas y métodos necesarios para la adquisición, asimilación e incorporación de nuevos conocimientos» que re- 
sulten en cambio técnico y que se reflejen en el desempeño de la empresa sin ser necesariamente innovaciones tecnológicas en sentido estricto (Tabla 2) (RICYT et al., 2001, pp. 37-38).

Tabla 2. Actividades de innovación Manual de Bogotá

Investigación y Desarrollo

Trabajo creativo sistemático para incrementar el conocimiento y su uso para crear nuevas aplicaciones (investigación básica, estratégica, aplicada, o de desarrollo experimental).

Fuente: RICYT et al. (2001 pp. 38-39).

Según la Encuesta de desarrollo e innovación tecnológica industria manufacturera -EDIT ${ }^{9}$ y la Encuesta de desarrollo e innovación tecnológica sector servicios y comercio -EDITS ${ }^{10}$ realizadas por el DANE, en 2014 dentro del grupo de organizaciones participantes de la ICGC las empresas no innovadoras representaron el 79,63\% para impresión y 71,13\% para edición (Figura 4).

Figura 4. DANE EDIT/EDITS Empresas por grado de innovación ICGC 2014

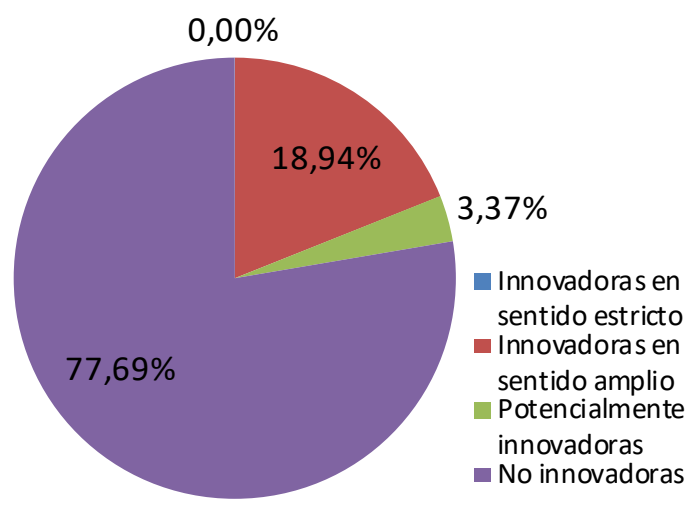

Fuentes: DANE (2015b, 2016b).

En 2014, la inversión en adquisición de maquinaria y equipo en las empresas de impre-

9 EDIT VII 2013-2014. 8.835 empresas industriales manufactureras (Impresión: CIIU Rev. 4 Agrupación industrial 181=481 empresas).

10 EDITS V 2014-2015. 8.056 empresas de sectores servicios y comercio (Edición: CIIU Rev. 4 Agrupación industrial 581=142 empresas).

\section{Esfuerzos de innovación}

Diseño, adquisición de tecnología (incorporada y no incorporada al capital), comercialización y capacitación. Acumulación de capital (físico, humano y de conocimiento e información) sión presentó una participación del 65,77\%. En las empresas de edición, la mayor inversión se realizó en TIC $(57,65 \%)$, seguido por adquisición de maquinaria y equipo $(18,82 \%)$ (Figura 5).

Figura 5. DANE EDIT/EDITS Monto invertido en ACTI, por tipo ICGC 2014

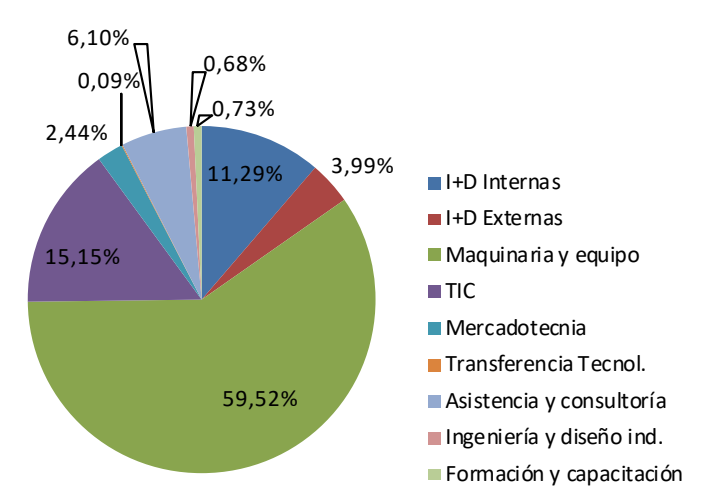

Fuentes: DANE (2015b, 2016b).

\subsection{INNOVACIÓN EN LA ICGC}

Las grandes empresas se mantienen y consolidan porque su ventaja se determina por la permanente posibilidad de acceso e incorporación de tecnología de punta, sumado a la vinculación de 
trabajadores especializados «que le permite responder con calidad y oportunidad a las exigencias de sus clientes». Sin embargo, las MiPyME no pueden alcanzar estos objetivos por los escasos recursos de capital y talento humano con los que cuen$\tan$ (SENA, 2013, p. 131).

Respecto a las fuentes de financiación de las MiPyME para procesos de innovación, y a los resultados sobre el acceso al crédito bancario, los programas del gobierno que fomentan la innovación «tienen baja cobertura e incidencia» (JUNCA Y MANRIQUE, 2014, p. 57).

De igual manera las PyME del sector «no están cualificadas para demandar conocimiento nuevo que les permita innovar y las instituciones no les ofrecen esa posibilidad» (FEDEGRÁFICA, 2014, p. 20).

Las cooperativas se constituyen en la respuesta a la necesidad de los pequeños empresarios de hacer frente a las relaciones con las grandes empresas y los proveedores de materias primas e insumos (SENA, 2013, p. 21).

Sin embargo, la Industria tiene «escasa tendencia grupal». Por tanto, esta situación dificulta el acceso de las microempresas a los beneficios de políticas de apoyo a la Industria y también la conformación de grupos sectoriales «con capacidad de influencia sobre las autoridades económicas para que estas arbitren políticas industriales ajustadas a las necesidades de renovación tecnológica en las que el sector gráfico se encuentra permanentemente inmerso» (FERRO Y GUISADO, 2010, pp. 7, 9).

Frente a estas situaciones, la interacción con el entorno es fundamental, pues favorece la capacidad de las organizaciones para saber interpretar las oportunidades y necesidades del mercado, a la vez que «modifica sus capacidades de aprendizaje, para detectar, interpretar, adaptar e incorporar nuevos conocimientos, afinando y potenciando sus competencias» (DINI Y STUMPO, 2011, p. 13).

\section{ACLARACIONES}

La caracterización de la ICGC que en 2012 realizó el equipo de investigadores del SENA-Cenigraf y del CID, contiene gran cantidad de información y análisis cuantitativo y cualitativo de la ICGC en varios aspectos, mas por el objetivo mismo de su investigación, su enfoque es la formación académica y para el trabajo de los integrantes de la ICGC.

De igual manera el trabajo realizado en 2015 por el PTP para evaluar el Plan de Negocios de la ICGC, contiene gran cantidad de información con énfasis en un análisis cuantitativo de la ICGC, mas su objetivo es precisamente evaluar el desempeño de la ICGC durante el periodo 2009-2014 y así replantear las estrategias según las nuevas condiciones de la ICGC y del entorno para alcanzar los objetivos del Plan de Negocios estructurado en 2009.

La EDIT (2013-2014) y EDITS (2014-2015) realizadas por el DANE a algunas organizaciones industriales de Colombia, contienen datos y cifras relevantes sobre tópicos de innovación. A juicio del investigador, por el diseño metodológico y la magnitud de estas encuestas, no alcanzan a incluir percepciones y opiniones de los empresarios participantes en cuanto al sector y su entorno.

Dichos trabajos son base importante para estructurar el estado del arte, sin embargo, en la etapa de documentación no se encontraron estudios anteriores con información reciente que profundizaran específicamente en el proceder de los 
empresarios frente a los cambios tecnológicos que impactan las prácticas tradicionales de la ICGC, en especial los que tienen que ver directamente con el proceso de impresión (núcleo de los procesos de producción de dicho sector).

\section{ASPECTOS METODOLÓGICOS}

Este estudio sigue las pautas de una investigación de tipo cualitativo para recolectar información sobre cómo las empresas de la muestra interactúan con su entorno y cómo los empresarios toman las decisiones para enfrentar los retos de actualización e innovación tecnológica del área de producción de su organización.

Este trabajo no utilizó lógica deductiva, pues no pretendía realizar generalizaciones sobre prácticas de gestión. Solamente aportar la identificación de ciertas prácticas de algunas organizaciones en determinada situación y contexto, para la comprensión de este fenómeno en particular. Se debe resaltar que la muestra es pequeña y que el análisis se realizó con base en la información recolectada de las intervenciones de los participantes, donde se recogen sus percepciones y opiniones sobre el tema.

Para realizar el análisis de la información de tipo cualitativo, en el desarrollo del presente trabajo se realizaron los subprocesos propuestos por Huberman y Miles (2000) (SALGADO, 2007, p. 74).

- Reducción de datos: Se seleccionaron con anticipación los aspectos relevantes del estado del arte, se agruparon por temas, se definieron las preguntas, se seleccionaron los participantes y se diseñaron los instrumentos para recolección de información.
- Presentación de datos: Se elaboraron las fichas por tema que contienen la información del subproceso anterior y se presentan en forma de cuadros estructurados.

\section{- Elaboración y verificación de conclusiones:}

Se señalaron los patrones de conceptos e ideas compartidas y temas recurrentes, así como similitudes y diferencias entre las opiniones de los participantes.

La estrategia de recolección de datos y análisis del objeto de estudio se fue adaptando según las condiciones que el investigador encontró en la realización del proyecto. Se diseñaron y rediseñaron las acciones propuestas y los instrumentos. Fue clave la crítica y consejos de investigadores especializados, que desde su experiencia en la realización de estudios sobre el sector ayudaron a dar nueva forma al método.

Los factores que influyeron en la decisión del rediseño de los instrumentos fueron: limitaciones de recurso (una sola persona coordinando contactos y aplicando instrumentos), baja capacidad de convocatoria (no es lo mismo que cite una institución a que lo haga un estudiante, así tenga el respaldo de la institución), y temas de logística (imposibilidad de coordinar única agenda de posibles participantes para aplicar instrumentos diseñados).

Se realizaron entrevistas semiestructuradas a personal de algunas organizaciones productivas (con planta de producción en alguna de las regiones con mayor presencia de la Industria en Colombia y que realizan procesos de impresión). De igual manera se incluyó en la muestra personal de algunas asociaciones, proveedores y entidades que apoyan el sector. 
El grupo focal debió migrar a entrevistas semiestructuradas. Se realizaron entrevistas a algunos expertos de la ICGC. La interacción y visión detallada de los procesos debió sustituirse, pues se percibió que los empresarios que participaron en las entrevistas semiestructuradas, si bien agendaron cita para colaborar con la entrevista, el tiempo que tenían era escaso y debían concentrarse en las actividades de su empresa. Se optó por participar como asistente a eventos de la ICG en Bogotá, donde se pudo de alguna manera conocer la percepción de los asistentes (dado que hay espacios para las preguntas del público y respuesta por parte de los expositores).

Cabe resaltar la buena voluntad de las personas que accedieron a participar en las entrevistas, de las personas que ayudaron haciendo nuevos contactos, y de las personas que accedieron a que se les presentara el proyecto y aportaron comentarios y críticas para darle forma. Esta colaboración es el recurso más valioso para la realización de este tipo de iniciativas independientes (y con restricción de recursos) que pretenden realizar un aporte al sector desde un proyecto de la academia.

Se realizaron en total 29 entrevistas semiestructuradas, distribuidas así: diez impresores, cuatro proveedores, dos cooperativas, tres entidades, y diez expertos del sector. Adicionalmente de los eventos especializados se extrajeron en total 14 exposiciones e intervenciones.

Finalmente, el total de documentos de la muestra (43) se distribuyeron así: asociaciones (5), entidades (6), impresores (10), proveedores (4), expertos (10), Clúster comunicación gráfica de Bogotá (4), proyectos Clúster comunicación gráfica de Bogotá (4). Como herramienta para análisis de la información cualitativa se utilizó el paquete RQDA ${ }^{11}$ de $R^{12}$.

Se aplicaron los instrumentos diseñados guardando los criterios para evaluar el rigor metodológico (SALGADO, 2007, p. 74).

- Dependencia: Durante el diseño de instrumentos se consultó con investigadores especializados con experiencia en la realización de estudios sobre el sector para verificar la propuesta de sistematización en la recolección y el análisis cualitativo del presente trabajo, buscando hacer un paralelo con investigaciones previas de características parecidas. En especial se destacan las reuniones con integrantes del grupo de trabajo encargado de elaborar la caracterización de la ICGC publicada en 2013 (funcionarios de SENA-Cenigraf e investigadores del Centro de Investigaciones para el Desarrollo (CID) de la Universidad Nacional de Colombia).

- Credibilidad: Se discutió con investigadores especializados con experiencia en la realización de estudios sobre el sector acerca de ejercicios previos para el contacto e interacción con los integrantes de la ICGC, con el fin de adaptar la estrategia para establecer los contactos para las entrevistas. Se decidió incluir entrevistas a otros integrantes de la ICGC con el fin de ampliar y complementar la información desde la perspectiva de otros participantes (diferentes a los impresores). Se transcribieron textualmente las entrevistas, y se incluyeron algunos fragmentos para respaldar las interpretaciones presentadas

\footnotetext{
11 Herramienta para análisis de información cualitativa. Software libre, multiplataforma.

12 Entorno y lenguaje de programación, empleado para cálculos estadísticos. Software libre, multiplataforma.
} 
en el documento final. Dichas interpretaciones se presentaron y discutieron con colegas diseñadores gráficos especializados en producción y con el profesor director del trabajo final.

- Auditabilidad: Se registraron y documentaron las decisiones del investigador en relación con el desarrollo y elaboración del trabajo final. Se describieron las características de los participantes y cómo se seleccionaron. Se realizaron grabaciones de audio de las entrevistas y se transcribieron textualmente.

- Transferibilidad: Se presentaron los elementos principales del contexto de la ICGC y se describieron las características de los participantes. Se señalaron los patrones de conceptos e ideas compartidas y temas recurrentes, así como similitudes y diferencias entre las opiniones de los participantes.

\section{CARACTERIZACIÓN DE PRÁCTICAS DE GESTIÓN DE ACTUALIZACIÓN E INNOVACIÓN TECNOLÓGICA EN ORGANIZACIONES DE LA ICGC}

A continuación se presenta el análisis de las respuestas al instrumento aplicado a diez impresores, complementado con el aplicado a cuatro proveedores y dos asociaciones.

\subsection{ORGANIZACIÓN}

En la muestra construida para el estudio, seis organizaciones tienen tradición familiar. Según Andigraf este elemento podría llegar a afectar la decisión de inversión cuando se prio- rizan las necesidades de la familia sobre las de la organización ${ }^{13}$.

En oferta de valor agregado resaltan tres organizaciones que han implementado diversas estrategias para atraer clientes: plataforma web-to-print, servicios de diseño para medios electrónicos, y diseño colaborativo con el cliente. Estos servicios adicionales a impresión se apoyan en los desarrollos de las TIC, así como en las industrias creativas.

\subsection{ENTORNO ORGANIZACIONAL}

Se identificó la tendencia de asociar a las instituciones académicas como fuente de mano de obra (ya sean empleados o pasantes) y no como posibles aliadas en proyectos de investigación o similares. Las principales alianzas y/o relaciones de los empresarios se establecen con proveedores y asociaciones.

Andigráfica ${ }^{14}$ aparece como el principal canal entre proveedores y empresarios (los participantes reconocen la feria como espacio para "hacer contactos"). Los proveedores exhiben su producto y dictan charlas que se programan en la agenda académica $^{15}$ del evento; los empresarios asisten para "estar al día" con tendencias y avances tecnológicos del sector.

En cuanto a relaciones con otras organizaciones del entorno, destaca la alianza de un impre-

\footnotetext{
13 Exposición Andigraf, en evento organizado por la SDE, septiembre 2017.

14 Feria nacional especializada, organizada por Andigraf cada dos años. 15 La estrategia de muchos proveedores es ofrecer como valor agregado servicios de capacitación, pero inevitablemente esta actividad es sesgada: se capacita en marcas y referencias y no en tecnología como tal. Por lo anterior podría existir cierto escepticismo respecto a este tipo de oferta.
} 
sor participante con la iniciativa Ruta $\mathrm{N}^{16}$, donde se aplican estrategias de empresas ancla: grandes empresas capacitan a las más pequeñas en temas de innovación.

\subsection{INVERSIÓN EN ACTI (ESPECÍFICAMENTE ADQUISICIÓN DE MAQUINARIA Y EQUIPO)}

La percepción de mejoras por actualización de maquinaria/equipo se resume en calidad, tiempos de respuesta, productividad y optimización de recursos. Dos organizaciones que atienden mercado internacional han realizado compra de maquinaria/equipo para impresión en los últimos cinco años con el objetivo de ser pioneras en tecnología en la ICGC.

Cuando son máquinas para impresión digital, los participantes prefieren comprarla nueva. En caso de maquinarias para acabados o de impresión offset, la decisión depende del tamaño de la empresa y su capital de trabajo. Cuando son inversiones altas, realizan la adquisición con recurso propio y financiación externa (leasing o crédito). No se percibió dificultad en el acceso a recursos externos.

Lo más usual es que la propuesta surja desde la organización (en especial del gerente en las PyME). A partir de una necesidad, buscan información sobre marcas y características principales de máquinas/equipos en fuentes disponibles (internet, ferias y publicaciones especializadas) y/o referencias de otras organizaciones del sector (experiencia con el servicio y máquinas de distintos proveedores).

16 Centro de innovación y negocios de Medellín, creado por la alcaldía de Medellín, UNE y EPM. Fuente: http://www.rutanmedellin.org/ es/20-preguntas-frecuentes/69-sobre-ruta-n
En la mayoría de los casos las negociaciones incluyeron instalación y puesta en marcha por parte del proveedor, debido a que es requisito para la garantía del fabricante. Se destacan dos organizaciones que realizan: capacitación "sobre la marcha" donde el operario recibe la instrucción sobre el uso de la máquina mientras ejecuta el proceso de producción; y preparación del personal con antelación a la capacitación del proveedor para "aprovechar mejor" el entrenamiento.

En general se encuentra que por ser equipos que integran sistemas de control electrónicos, ya no es común que el empresario realice adaptaciones de componentes (como sí era práctica usual con las máquinas de impresión antiguas). Tres organizaciones usan sustratos ${ }^{17}$ diferentes a los recomendados por el fabricante, y/o realizan cambios en los ajustes de configuración de la máquina para manipular dichos sustratos.

\subsection{GESTIÓN DE ACTUALIZACIÓN E INNOVACIÓN TECNOLÓGICA}

Seis organizaciones incluyen instancias adicionales a la gerencia en la toma de decisiones sobre inversión en proyectos de actualización e innovación tecnológica. De este grupo hay reconocimiento formal de equipos de gestión de innovación o similares en dos organizaciones. Sobre el nivel de formación del personal que participa en estas actividades, se encuentra que la mayoría son profesionales y pertenecen a cargos altos y medios de las organizaciones. Resaltan dos organizaciones que incluyen en algunos proyectos la opinión y concepto de operarios.

17 Soporte que recibe la tinta en el proceso de impresión: papel, plástico, tela, etc. 
Para aprobación de proyectos de actualización e innovación tecnológica, en cuatro organizaciones la decisión final la toma el gerente. En las otras seis, es una decisión conjunta, donde además se identifican procedimientos formales para la evaluación y aprobación de proyectos de inversión. Respecto a los criterios que se tienen en cuenta para la toma de decisiones, se resumen en que se necesite la adquisición, que exista el recurso y que traiga mejoras para la organización.

El apoyo desde la administración para temas de capacitación sobre innovación o gestión, se resume en garantizar el tiempo para que estas personas puedan asistir a los lugares que ofrecen esta formación, pues en general se trata de convocatorias a eventos o cursos que no tienen un costo significativo (por ej. cursos del SENA, agenda académica de las ferias especializadas, etc.). Sobresale una organización que tiene la innovación como estrategia competitiva, por lo que la administración facilita la formación a sus empleados en temas relacionados, desde la gerencia hasta el nivel operativo.

En las organizaciones participantes, lo más usado como canal para recibir y transmitir las sugerencias del personal sobre actualización e innovación tecnológica es el contacto directo de la gerencia con los niveles operativos, o seguir el conducto regular. Se destaca una organización que emplea estrategias más estructuradas para motivar a los empleados a participar en iniciativas de promoción de la innovación y aportar ideas que ayuden a la organización.

\section{FACTORES QUE AFECTAN E INFLUYEN LAS PRÁCTICAS DE GESTIÓN DE ACTUALIZACIÓN E INNOVACIÓN TECNOLÓGICA EN ORGANIZACIONES DE LA ICGC}

A continuación se presenta el análisis de las respuestas al instrumento aplicado a diez expertos del sector, complementado con el aplicado a los otros participantes, e intervenciones en algunos eventos de la Industria. Se destacaron dos factores: obstáculos y alianzas.

\subsection{OBSTÁCULOS QUE HAN AFECTADO A EMPRESARIOS Y ORGANIZACIONES DE LA ICGC}

- Fallas en políticas diseñadas desde el Estado para promover la innovación en la Industria, donde falta continuidad, hay exceso de trámites (burocracia), y dificultad en el acceso a las MiPyME.

- Falta de acompañamiento al empresario desde un ente que cumpla las funciones de un Centro de Desarrollo Tecnológico $\left(\mathrm{CDT}^{18}\right)$ de la Industria19 20 .

- Baja integración con industrias TIC y creativas, cuya posible explicación sea el "temor al cam-

18 «Organizaciones públicas o privadas, dedicadas al desarrollo de proyectos de investigación aplicada, el desarrollo de tecnología propia y actividades de transferencia» Fuente: http://www.colciencias.gov.co/ portafolio/reconocimiento_de_actores/centros-desarrollo-tecnologico 19 Andigraf con su "Coordinación de mejoramiento continuo e innovación" hizo el esfuerzo de retomar desde finales de 2015 la labor que realizaba Cigraf, pero al parecer todavía no logra el reconocimiento entre los integrantes de la Industria.

20 El Centro de desarrollo tecnológico para la competitividad de la industria de la comunicación gráfica (Cigraf), fue creado en 1991 como Instituto para la formación y el desarrollo tecnológico de la industria gráfica (Iftag). En 2001 se constituyó como fundación y funcionó hasta 2014 
bio, a lo desconocido" por parte del empresario del sector.

- Entre los empresarios de la Industria no es fuerte la visión de trabajo conjunto con pares y otros integrantes del entorno, además hay baja conciencia sobre la importancia de gestionar e invertir en innovación (individual o colectivamente) ${ }^{21}$.

- Baja demanda del producto impreso por cambios en la distribución de los presupuestos para publicidad de las industrias cliente ${ }^{22}$, y por estrategias de greenwashing ${ }^{23}$ que desestimulan el uso de productos fabricados con papel.

- Factores que afectan el flujo financiero y también la disponibilidad de recurso para inversión de la organización: dependencia de tasa de cambio (alto nivel de importación de insumos, que deriva en incremento de costos de producción), "negociación dura" por parte de industrias cliente (prioridad precio y ampliación de plazos de pago), entre otros. Así mismo, estos factores conllevan baja rentabilidad.

- Prácticas informales como estrategia de algunas empresas del sector para bajar estructura de costos y competir en la "guerra de precios". Esto se relaciona con la percepción de falta de control por parte del Estado para combatir este factor que afecta a las organizaciones que optan por realizar su operación dentro de la formalidad.

- Si bien subyace la necesidad de realizar actualización tecnológica, los empresarios no tienen

21 Se expone como ejemplo la desaparición de Cigraf.

22 La ICG se considera transversal (hace parte de otras cadenas de valor) al ofrecer servicios a diversos sectores económicos: farmacéutico, cosmético, alimentos, entre otros.

23 Se refiere a prácticas de algunas empresas donde se "sataniza" el papel, como estrategia "errónea" para promocionar lo digital sobre lo impreso. El término "greenwashing" fue acuñado en los 80's por el ecologista Jay Westerveld. Fuente: https://www.theguardian.com/sustainable-business/2016/aug/20/greenwashing-environmentalism-lies-companies motivación para realizarla debido a altos niveles de subutilización de bienes de capital sumado a percepción de lento retorno de la inversión. Esto es debido a factores como altos costos para renovación tecnológica (versus los bajos aranceles para importación de maquinaria usada), pues existe el riesgo de realizar altas inversiones y que organizaciones informales compitan con maquinaria usada.

\subsection{ALIANZAS QUE PODRÍAN INFLUIR EL AVANCE Y DESARROLLO DE LA ICGC}

- Entre el empresario y sus colaboradores: Fortalecer el vínculo con los trabajadores de las organizaciones mediante programas de capacitación y formación, y crear ambientes que estimulen la innovación, donde fluya la información y las ideas.

- Entre la organización y sus clientes: Establecer dinámicas de trabajo colaborativo con los clientes para desarrollo de productos y procesos de producción.

- Entre la organización y sus proveedores: Establecer dinámicas de negociación con los proveedores de la Industria donde se garanticen condiciones favorables para el desarrollo de productos y procesos.

- Entre la organización e instituciones académicas: Establecer dinámicas de investigación conjunta y de trabajo colaborativo con instituciones académicas para el desarrollo de productos y procesos de producción.

- Entre organizaciones del sector: Fortalecer mecánicas de asociación y colaboración entre pares para dinamizar la gestión de actualización 
e innovación tecnológica de las organizaciones y facilitar el acceso de estas a recursos del Estado que promueven el desarrollo de la Industria.

- De la ICGC con industrias TIC y creativas: Implementar dinámicas de investigación conjunta y de trabajo colaborativo para desarrollo de productos y procesos de producción.

- Integración cadena de valor: Configuración como cadena de los integrantes de la Industria, para incrementar el valor agregado y potenciar las dinámicas de negociación, producción y comercialización.

- Conformación de clúster: Participación e interacción de los integrantes de la Industria, para generar y promover iniciativas que impacten la competitividad, productividad e innovación de las organizaciones del sector.

\section{POLÍTICAS DE APOYO A LA ICGC PARA GESTIÓN DE ACTUALIZACIÓN E INNOVACIÓN TECNOLÓGICA}

A continuación se exponen los factores identificados durante la aplicación del instrumento a tres entidades, complementado con intervenciones en algunos eventos de la Industria.

\subsection{ALCALDÍA DE BOGOTÁ. SECRETARÍA DE DESARROLLO ECONÓMICO (SDE)}

La SDE asume que apoyando el desarrollo de la economía naranja ${ }^{24}$, se beneficiaría también a la Industria. Ha enfocado su labor en las aglomeracio-

24 La definición de economía creativa de John Howkins se sintetiza como la que «comprende los sectores en los que el valor de sus bienes y servicios se fundamenta en la propiedad intelectual» (BUITRAGO Y DUQUE, 2013, p. 15). nes de la ciudad, y para el caso específico de la ICG actúa en el Ricaurte. Mediante recorridos a los territorios se acercan a los empresarios del sector para saber de primera mano cuáles son sus necesidades. A partir de estos escenarios de diálogo con los empresarios trabajan en conjunto con la alcaldía local y el edil de la localidad para realizar mesas de trabajo.

\subsection{MINISTERIO DE COMERCIO, INDUSTRIA Y TURISMO (MINCIT). DIRECCIÓN DE PRODUCTIVIDAD Y COMPETITIVIDAD}

Desde el MinCIT se tiene como guía de acción la Política de Desarrollo Productivo para todas las industrias del país. Una de las líneas de acción es innovación y emprendimiento ${ }^{25}$, con el fin de lograr altos niveles de sofisticación y diversificación de la economía en Colombia. Realiza acciones de acompañamiento, facilitando los relacionamientos con los órganos del gobierno nacional y territorio, con el fin de que la Industria pueda aprovechar de mejor manera los instrumentos de política diseñados desde el Estado para mejorar las condiciones de la Industria y el empresariado.

\subsection{SENA. CENTRO PARA LA INDUSTRIA DE LA COMUNICACIÓN GRÁFICA (CENIGRAF)}

Cenigraf es el centro especializado donde se capacita y certifica en competencias laborales específicas del sector. Está en contacto permanente con la Industria para evaluar y actualizar sus programas de formación según las necesidades de las organi-

25 Dentro de las estrategias y acciones de esta política se encuentra: «Línea de acción 2: Aumentar la actividad innovadora y el emprendimiento en el aparato productivo» (CONPES, 2016, pp. 73-75). 
zaciones. Esto conecta además con la "formación para el trabajo" del SENA, donde la empresa hace parte importante en la adquisición de competencias laborales del aprendiz, a través del contrato de aprendizaje. Están trabajando también con formación de semilleros y grupos de investigación.

Desde hace algunos años, Cenigraf ha ampliado su oferta de programas de formación para incluir a las industrias creativas. Por ejemplo: formación tecnológica en producción multimedia, animación 3D, entre otros ${ }^{26}$.

\section{CONSIDERACIONES FINALES}

A continuación se recogen los principales elementos que a partir de la información recolectada y presentada en secciones anteriores, permiten avanzar una caracterización de las prácticas de gestión de los procesos de actualización e innovación tecnológica en el área de producción de las organizaciones de la ICG en Colombia.

Las prácticas a destacar de las empresas participantes son:

- Ofrecer servicios adicionales a impresión (apoyándose en los desarrollos de las TIC y las industrias creativas).

- Aprovechar de mejor manera la capacitación del proveedor (entrenamiento "sobre la marcha" y preparación con antelación).

- Establecer alianzas con organizaciones donde específicamente se promueva la innovación basada en tecnología para incrementar la competitividad.

- Reconocimiento formal de equipos de gestión de la innovación (conformación y respaldo).

- Implementar procedimientos formales para ges- tión de proyectos de actualización e innovación tecnológica (estructuración, evaluación, aprobación, implementación, retroalimentación).

- Apoyar desde la administración la capacitación al personal administrativo y operativo en temas de gestión y de innovación (inversión de tiempo y capital).

- Ejecutar estrategias de motivación y entrega de incentivos que promuevan generación y aporte de ideas para innovación en la organización.

Los obstáculos del entorno identificados son:

- Fallas en políticas diseñadas desde el Estado para promover la innovación en la Industria (dificultad acceso, falta de continuidad).

- Ausencia de un CDT de la Industria (asesoría y acompañamiento).

- Incertidumbre del comportamiento económico del sector (dependencia tasa de cambio, baja demanda producto impreso, "negociación dura" por parte de industrias cliente, "guerra de precios", entre otros).

- Baja asociatividad y poco trabajo conjunto con integrantes del entorno.

Las alianzas entre integrantes de la Industria y entidades de apoyo deberían promover entre los participantes:

- Dinámicas de negociación favorable.

- Dinámicas de trabajo colaborativo y/o investigación (avances conjuntos).

- Mecánicas de asociación y colaboración (facilitar acceso a recursos del Estado).

- Integración cadena de valor (potenciar negociación, producción y comercialización).

- Conformación de clúster (interacción para generar y promover iniciativas de mejora en competitividad, 
productividad e innovación).

En el análisis se encontró que las políticas de apoyo a la Industria diseñadas desde el Estado incluyen factores de formación y financiación, pero se hace énfasis en la articulación del sector con los organismos del sector público o privado que puedan apoyarlas en el mejoramiento de la competitividad. Así mismo, se encontró que el enfoque está ampliándose para incluir no solo a la ICGC sino a las que se enmarcan en la economía naranja (por ejemplo industrias creativas).

De igual manera, que las asociaciones constituyen la oportunidad para muchos empresarios de poder presentarse a convocatorias de programas de apoyo a la Industria diseñados por el Estado. Sobre todo porque el contacto de las entidades no es "directo" con el empresario, sino a través de organizaciones que los agrupen y representen.

Se espera que el presente ejercicio de caracterización permita la identificación de modos de proceder de las organizaciones y de factores que las afectan, y que sirva de insumo para el diseño de políticas, de iniciativas o estrategias de organismos públicos o privados que apoyan el sector. Específicamente donde se promueva la formalización e implementación de la gestión de la innovación en las organizaciones de la Industria, en especial de las MiPyME que por tener escasez de recursos deben sortear los obstáculos del entorno con mayor dificultad.

Así mismo que los elementos presentados puedan despertar el interés en los empresarios para solicitar a estos organismos asesoría y acompañamiento para realizar esta labor, pues, aunque se reconozca la importancia de la gestión de la innovación, no existen "fórmulas mágicas" para implementarla. Cada organización requiere herramientas ajustadas a los propósitos, características, y necesidades propias.

\section{ALGUNAS RECOMENDACIONES}

Se presentan a continuación propuestas que podrían favorecer el desarrollo de estrategias de apoyo al sector.

\subsection{ACCESO A LA INFORMACIÓN}

No todas las organizaciones tienen la disponibilidad de asignar recursos para pagar consultorías o acceder a estudios económicos, por lo que al menos garantizar fácil acceso a información especializada les permitiría plantear y planear mejor sus estrategias. Con libre flujo de información, el análisis, procesamiento y aprovechamiento queda en manos de cada organización.

Por otro lado, en charlas con investigadores especializados con experiencia en la realización de estudios sobre el sector, se tocó el tema de la dificultad de acceso a información actualizada sobre la Industria, así como la falta de unidad en los criterios de presentación de la información, derivado también de las múltiples instancias de las que se origina y de las otras tantas que procesan la información.

En este sentido la iniciativa del observatorio de la Industria, promovida por el Clúster de Comunicación Gráfica en Bogotá y liderada por Uniminuto ${ }^{27}$, es un esfuerzo que podría traer muchos beneficios a las organizaciones del sector (en especial a las MiPyME), y a investigadores. El poder tener información centralizada, de distintas fuentes (organismos del sector público y privado), organi-

27 Corporación Universitaria Minuto de Dios. 
zada y procesada con un criterio determinado, es un factor clave.

\subsection{MÁS TIPOS (OPCIONES) DE ASOCIACIÓN}

Para ser parte de Andigraf o de las cooperativas se les exige a los empresarios tener formalizada su empresa y realizar aportes de capital. Si la empresa no puede (o no desea) cumplir estas condiciones ¿por qué no probar otro tipo de figura de asociación? Por ejemplo, junta comunal, o consejo empresarial. El objetivo es fortalecer las alianzas entre pares y trabajar en busca de un beneficio común, además de tener posibilidad de acceso a los recursos de las políticas de apoyo a la Industria diseñados por el Estado.

Se sugiere a los organismos públicos o privados que apoyan el sector, promover espacios de encuentro, de discusión y de apoyo a empresarios que tal vez en el momento no estén en ningún tipo de asociación, para que también puedan percibir y conocer los beneficios que trae el trabajar en equipo por el beneficio común.

\subsection{OPORTUNIDADES ECONOMÍA NARANJA}

Las organizaciones de la ICGC están Ilamadas a beneficiarse del auge que vive la economía naranja y de todos los desarrollos que se están generando alrededor de esta, sobre todo en tema de políticas que se están diseñando e implementando desde el Estado. Por tanto, establecer y fortalecer alianzas con organizaciones e instituciones de las otras categorías de actividades del "universo naran- ja"28, aprovechando no solamente su posición de "intersección", sino la gran riqueza de nuestro país, fuente de talento creativo y patrimonio cultural.

De igual manera, las organizaciones del sector deben orientar esfuerzos a la realización de proyectos de colaboración y de negocios que puedan hacer uso de la infraestructura ya establecida de la ICGC (propia de una industria madura, con recursos y amplia experiencia de atención a otras industrias), y que también faciliten posibles acercamientos e integración con industrias que están cercanas a las industrias creativas, tal como lo es la industria TIC.

\subsection{AGENDA CONJUNTA Y DIFUSIÓN}

Es interesante la labor del Clúster de Comunicación Gráfica de Bogotá que actúa como facilitador para centralizar la agenda de formación académica que las instituciones ofrecen al sector. Se reconoce la posibilidad de que múltiples actividades se crucen en horarios y/o fechas, pero las organizaciones tienen disponible la programación para elegir a qué evento asistir.

Se sugiere a los organismos públicos o privados que apoyan el sector, replicar en otros temas este esquema de trabajo de "agenda unificada", como herramienta de planeación para el empresario. Es decir, que se sumen más organizaciones, instituciones y entidades a esta dinámica de integración y difusión.

\section{Lista de abreviaturas}

28 «El universo naranja está compuesto por: i) la Economía Cultural y las Industrias Creativas, en cuya intersección se encuentran las Industrias Culturales Convencionales; y ii) las áreas de soporte para la creatividad» (BUITRAGO Y DUQUE, 2013, p. 40). 
- ACTI Actividades de ciencia, tecnología e innovación

- Andigraf Asociación colombiana de la industria de la comunicación gráfica

- CID Centro de Investigaciones para el Desarrollo. Universidad Nacional de Colombia

- CDT Centro de desarrollo tecnológico

- DANE Departamento administrativo nacional de estadística

- EAM Encuesta anual manufacturera

- EDIT Encuesta de desarrollo e innovación tecnológica industria manufacturera

- EDITS Encuesta de desarrollo e innovación tecnológica sector servicios y comercio

- I+D Investigación y desarrollo

- ICG Industria de la comunicación gráfica

- ICGC Industria de la comunicación gráfica en Colombia

- MiPyME Micro, pequeñas y medianas empresas

- MinCIT Ministerio de comercio, industria y turismo

- OCDE Organización para la cooperación y el desarrollo económicos

- OCyт Observatorio colombiano de ciencia y tecnología

- PIB Producto interno bruto

- PTP Programa de transformación productiva

- RICYT Red de indicadores de ciencia y tecnología -iberoamericana e interamericana-

- SENA Servicio nacional de aprendizaje

- TACC Tasa de crecimiento anual compuesto

- TIC Tecnologías de la información y la comunicación

\section{REFERÊNCIAS}

ANDIGRAF (1994) Procesos en la Industria Gráfica. Asociación Colombiana de Industrias Gráficas. Bogotá, Colombia.

ANDIGRAF (2017). Boletín Económico. Diciembre 2016. Asociación Nacional de la Industria de la Comunicación Gráfica. Bogotá, Colombia. Disponible: http://www.andigraf.com.co/sites/default/files/informe_cierre_de_ano2016.pdf
BRICEÑO, M. (2012). Caracterización de la cadena de abastecimiento en las Pymes de la Comunicación Gráfica Impresa en Colombia. Trabajo de monografía para optar el título de Ingeniero de Producción. Facultad de Ingeniería. Universidad EAN. Bogotá, Colombia. Disponible: http://repository.ean.edu.co/ bitstream/handle/10882/1582/BricenoManuel2012.pdf 


\section{REFERÊNCIAS}

BUITRAGO, F. y DUQUE, I. (2013) La Economía Naranja, una oportunidad infinita. Banco Interamericano de Desarrollo. Disponible: https:// publications.iadb.org/handle/11319/3659

CONSEJO NACIONAL DE POLÍTICA ECONÓMICA Y SOCIAL (2016). Política nacional de Desarrollo Productivo. Conpes 3866. Departamento Nacional de Planeación. República de Colombia. Disponible: https:// colaboracion.dnp.gov.co/CDT/Conpes/ Económicos/3866.pdf

DANE (2010-2016) Encuesta Anual Manufacturera. Años 2009-2015. Departamento Administrativo Nacional de Estadística. Presidencia de la República de Colombia. Disponible: http://www.dane.gov.co/index.php/estadisticas-por-tema/industria/encuesta-anual-manufacturera-enam

DANE (2015) Encuesta de Desarrollo e Innovación Tecnológica 2013-2014 Industria manufacturera. Departamento Administrativo Nacional de Estadística. Presidencia de la República de Colombia. Disponible: https://www.dane.gov.co/ files/investigaciones/boletines/edit/anexo_EDIT_ Manufacturera_2013_2014.xls

DANE (2016) Encuesta de Desarrollo e Innovación Tecnológica 2014-2015 Sector Servicios y Comercio. Departamento Administrativo Nacional de Estadística. Presidencia de la República de Colombia. Disponible: https://www.dane. gov.co/files/investigaciones/boletines/edit/Anexo_ EDIT_Servicios_2014_2015.xls
DANE (2017) Colombia, exportaciones totales, según CIIU Rev. 3 1995-2017. Departamento Administrativo Nacional de Estadística. Presidencia de la República de Colombia. Disponible: https://www. dane.gov.co/files/investigaciones/comercio_exterior/exportaciones/2017/anexos_export_ene17.xls

\section{DANE (2017) Cuentas Trimestrales-Colombia} Producto Interno Bruto (PIB) Cuarto Trimestre de 2016. Departamento Administrativo Nacional de Estadística. Presidencia de la República de Colombia. Disponible: https://www.dane.gov.co/ files/investigaciones/boletines/pib/Anexos_oferta_ constantes_desestacionalizadas_IV_2016.xls

DINI, M. y STUMPO, G. (2011) Políticas para la innovación en las pequeñas y medianas empresas en América Latina. Colección Documentos de proyectos. Comisión Económica para América Latina y el Caribe. Disponible: http://www.cepal. org/es/publicaciones/3868-politicas-la-innovacion-pequenas-medianas-empresas-america-latina

FEDEGRÁFICA (2014) ¿Cómo innovar en pymes gráficas? Dimensión gráfica (36), diciembre, pp. 19-22. Federación Colombiana de Cooperativas de Empresarios de la Industria de la Comunicación Gráfica. Bogotá, Colombia. Disponible: http:// www.fedegrafica.com.co/revista

FERRO, C. y GUISADO, M. (2010). Análisis de la Industria Gráfica en el contexto del sector manufacturero español. Revista Galega de Economía, 19 (2), pp. 1-18. Facultade de Ciencias Económicas e Empresariais, Universidade de Santiago de Compostela. Disponible: http:// umw.usc.es/econo/RGENol19_2/castelan/art8c.pdf 


\section{REFERÊNCIAS}

GENTLEMAN R. e IHAKA, R. (1997) R. Environment for statistical computing and graphics. GNU license. Disponible: https://www.r-project.org/ HUANG, R. (2016). RQDA: R-based Qualitative Data Analysis. $R$ package version 0.2-8. BSD license. Disponible: http://rqda.r-forge.r-project.org/

JUNCA, G. y MANRIQUE, O. (2014). Caracterización e identificación de aglomeraciones y cadenas productivas en los sectores de industria y servicios en la ciudad de Bogotá. Cuadernos de Desarrollo Económico (26). Subdirección Estudios Estratégicos. Dirección de Estudios Socioeconómicos y Regulatorios. Secretaría de Desarrollo Económico. Alcaldía Mayor de Bogotá. Bogotá, Colombia. Disponible: http://observatorio.desarrolloeconomico.gov.co/directorio/documentosPortal/Cuaderno26.pdf

MALAVER, F. (2002). Las transformaciones empresariales en la industria de las artes gráficas. Los retos analíticos de las historias por contar. INNOVAR Revista de Ciencias administrativas y sociales, (19), pp. 31-48. Universidad Nacional de Colombia. Bogotá, Colombia. Disponible: http:// www.bdigital.unal.edu.co/26378/

MALAVER, F. (2002). Un perfil de las capacidades tecnológicas en la industria de artes gráficas, imprentas y editoriales. INNOVAR Revista de Ciencias Administrativas y Sociales, (20); pp. 5581. Universidad Nacional de Colombia. Bogotá, Colombia. Disponible: http://www.bdigital.unal. edu.co/26654/
MALAVER, F. y VARGAS, M. (2012) Luces y sombras del vínculo entre el diseño y la innovación industrial. INNOVAR Revista de Ciencias Administrativas y Sociales, (22) 46; pp. 149-164. Universidad Nacional de Colombia. Bogotá, Colombia. Disponible: http://www.bdigital.unal.edu.co/37528/

MALAVER, F. y VARGAS, M. (2013) Formas de innovar y sus implicaciones de política: Lecciones de una experiencia. Cuadernos de Economía, 32 (60), pp. 537-570. Facultad de Ciencias Económicas, Universidad Nacional de Colombia. Bogotá, Colombia. Disponible: http://www.bdigital.unal.edu.co/37930/

MUNIVE, M. (2015) Detonadores de la modernización tecnológica en la industria gráfica de México: una metodología y un caso de éxito. Ingeniería Investigación y Tecnología, XVI (3), pp. 317-334. Universidad Nacional Autónoma de México. México D.F., México. Disponible: http:// www.scielo.org.mx/pdf/iit/v16n3/v16n3a1.pdf

OCDE (2006). Manual de Oslo. Guía para la recogida e interpretación de datos sobre innovación. Tercera edición. Organización de Cooperación y Desarrollo Económicos; Oficina de Estadísticas de las Comunidades Europeas. Disponible: http://www.oecd-ilibrary.org/science-and-technology/manual-de-oslo_9789264065659-es

PÉREZ, R.; DÍAZ, H. y VARGAS, H. (2014). Gestión en siete PyMEs de la comunicación gráfica. Revista EAN, Escuela de Administración de Negocios, (76), pp. 174-192. Bogotá, Colombia. Disponible: http://journal.ean.edu.co/index.php/Revista/article/view/804/769 


\section{REFERÊNCIAS}

PTP (2009) Desarrollando sectores de clase mundial en Colombia. Informe Final Sector Industria de la Comunicación Gráfica. Programa de Transformación Productiva, Ministerio de Comercio, Industria y Turismo. McKinsey \& Company. Bogotá, Colombia. Disponible: https:// www.ptp.com.co/documentos/Plan de Negocios Industria Comunicacion Grafica.pdf

PTP (2013). Estudio para definir y caracterizar la informalidad en 8 sectores seleccionados del Programa de Transformación Productiva. Sector Industria Editorial y de la Comunicación Gráfica. Resumen Ejecutivo. Programa de Transformación Productiva. Bancóldex y Ministerio de Comercio, Industria y Turismo. Universidad Sergio Arboleda. Bogotá, Colombia. Disponible: https://www.ptp.com.co/documentos/ Resumen_Ejecutivo_IG_VF.pdf

PTP (2016) Evaluación del Plan de Negocios del Sector Industria de la Comunicación Gráfica. Producto 1: Informe de Resultados de la Evaluación de Desempeño del Sector Industria Editorial y de la Comunicación Gráfica. Período: 2009-2014. Programa de Transformación Productiva, Bancóldex y Ministerio de Comercio, Industria y Turismo. Universidad EAN. Bogotá, Colombia. Disponible: https://www.ptp.com.co/documentos/ Entregable 1 -Ind Ed actualizado.pdf

PTP (2016) Producto 3: Parte 1: Análisis Macro Económico y de Competitividad de la Industria de la Comunicación Gráfica. Programa de Transformación Productiva, Bancóldex y Ministerio de Comercio, Industria y Turismo. Universidad
EAN. Bogotá, Colombia. Disponible: https://www. ptp.com.co/documentos/Entre 3 - Ind Ed.pdf

PTP (2016) Evaluación del Plan de Negocios del Sector Industria de la Comunicación Gráfica. Producto 5: Propuesta de Seguimiento y Control del Plan de Negocios de la Industria Editorial y de la Comunicación Gráfica. Programa de Transformación Productiva, Bancóldex y Ministerio de Comercio, Industria y Turismo. Universidad EAN. Bogotá, Colombia. Disponible: https://www.ptp. com.co/documentos/Propuesta de Seguimiento y Control PdN I.G. V.F.Entregable 5.pdf

RICYT (2001) Manual de Bogotá. Normalización de Indicadores de Innovación Tecnológica en América Latina y el Caribe. Red Iberoamericana e Interamericana de Indicadores de Ciencia y Tecnología, Organización de Estados Americanos, Programa iberoamericano de ciencia y tecnología para el Desarrollo, Colciencias y Observatorio de Ciencia y Tecnología. Disponible: http://www.ricyt.org/ manuales/doc_view/5-manual-de-bogota

ROBAYO, P. (2015). La innovación como proceso y su gestión en la organización: una aplicación para el sector gráfico colombiano. Trabajo de grado para obtener el título de Magíster en Administración.Facultad de Ciencias Económicas, Universidad Nacional de Colombia. Bogotá, Colombia. Disponible: http://www.bdigital.unal.edu.co/51128/

SALGADO, A. (2007) Investigación cualitativa: diseños, evaluación del rigor metodológico y retos. Liberabit. Revista de Psicología, (13), pp. 7178. Universidad de San Martín de Porres. Lima, 


\section{REFERÊNCIAS}

Perú. Recuperado de: http://www.redalyc.org/articulo.oa?id=68601309

SANTAMARÍA, L.; NIETO, M. y BARGE-GIL, A. (2009) ¿Hay innovación más allá de la I+D? El papel de otras actividades innovadoras. Universia Business Review, (22), pp. 102-117. Universia. España. Disponible: www.redalyc.org/ pdf/433/43311704007.pdf
SENA (2013). Estudio de Caracterización Ocupacional de la Industria de la Comunicación Gráfica en Colombia. Servicio Nacional de Aprendizaje. Universidad Nacional de Colombia. Facultad de Ciencias Económicas. Centro de Investigación para el Desarrollo. Bogotá, Colombia. Disponible: http://comunicaciongraficasena.blogspot.com.co/p/mesa-sectorial-artes-graficas.html 